\title{
Formation of a Motor Memory by Action Observation
}

\author{
Katja Stefan, ${ }^{1,3}$ Leonardo G. Cohen, ${ }^{1}$ Julie Duque, ${ }^{1}$ Riccardo Mazzocchio, ${ }^{1}$ Pablo Celnik, ${ }^{1}$ Lumy Sawaki, ${ }^{1}$ \\ Leslie Ungerleider, ${ }^{2}$ and Joseph Classen ${ }^{3}$ \\ ${ }^{1}$ Human Cortical Physiology Section, National Institute of Neurological Disorders and Stroke-National Institutes of Health (NIH), Bethesda, Maryland \\ 20892, ${ }^{2}$ Laboratory of Brain and Cognition, National Institute of Mental Health-NIH, Bethesda, Maryland 20892, and ${ }^{3}$ Human Cortical Physiology and \\ Motor Control Laboratory, Department of Neurology, University of Würzburg, 97080 Würzburg, Germany
}

\begin{abstract}
Mirror neurons discharge with both action observation and action execution. It has been proposed that the mirror neuron system is instrumental in motor learning. The human primary motor cortex (M1) displays mirror activity in response to movement observation, is capable of forming motor memories, and is involved in motor learning. However, it is not known whether movement observation can lead directly to the formation of motor memories in the M1, which is considered a likely physiological step in motor learning. Here, we used transcranial magnetic stimulation (TMS) to show that observation of another individual performing simple repetitive thumb movements gives rise to a kinematically specific memory trace of the observed motions in M1. An extended period of observation of thumb movements that were oriented oppositely to the previously determined habitual directional bias increased the probability of TMS-evoked thumb movements to fall within the observed direction. Furthermore, the acceleration of TMS-evoked thumb movements along the principal movement axis and the balance of excitability of muscle representations active in the observed movements were altered in favor of the observed movement direction. These findings support a role for the mirror neuron system in memory formation and possibly human motor learning.
\end{abstract}

Key words: action observation; motor cortex; human; plasticity; mirror neuron system; memory

\section{Introduction}

Although most motor skills are acquired through physical practice, the mere observation of movements has also been shown to lead to subsequent specific performance gains (Vogt, 1995; Black and Wright, 2000; Brass et al., 2001; Heyes and Foster, 2002; Horn et al., 2002; Vinter and Perruchet, 2002; Edwards et al., 2003; Petrosini et al., 2003; Mattar and Gribble, 2005). There is strong evidence from studies involving the physical practice of movements that an important physiological component of behavioral performance gains is a lasting change of local cortical movement representations, a kind of motor memory (Karni et al., 1995; Pascual-Leone et al., 1995; Kleim et al., 1998; Sanes and Donoghue, 2000; Conner et al., 2003). However, it is unknown whether observation alone is sufficient to leave a memory trace in movement representations.

A priori, the formation of local motor memories by observation of movements would appear to be a highly unlikely event, given the immense computational problem of transforming the

\footnotetext{
Received June 4, 2005; revised Aug. 9, 2005; accepted Aug. 28, 2005.

This work was supported in part by the Intramural Research Program of the National Institute of Neurological Disorders and Stroke-National Institutes of Health, by a grant from the Akademie der Naturforscher LEOPOLDINA to K.S. (Germany BMBF-LPD 9901/8-50), by the Hertie-Foundation, Germany, and by the State of Bavaria, Germany We are grateful to Steven $P$. Wise for helpful comments.

Correspondence should be addressed to either of the following: Dr. Joseph Classen, Department of Neurology, University of Wuerzburg, Josef-Schneider Strasse 12, 97080 Wuerzburg, Germany, E-mail: Classen__@klinik.uniwuerzburg.de; or Dr. Leonardo G. Cohen, Human Cortical Physiology Section, National Institute of Neurological Disorders and Stroke-National Institutes of Health, Building 10, Room 5N226, Bethesda, MD 20817, E-mail: cohen!@ninds.nih.gov.

DOI:10.1523/JNEUROSCI.2282-05.2005

Copyright $\odot 2005$ Society for Neuroscience $\quad$ 0270-6474/05/259339-08\$15.00/0
}

visual representation of movements input into a congruent motor output. However, recently, in several brain areas of the macaque monkey, neurons have been discovered that discharge both when the monkey performs a specific action and when it observes a similar action performed by another agent (Rizzolatti and Craighero, 2004). These neurons have been termed "mirror neurons” (Gallese et al., 1996). Given its astonishing properties, it has been proposed that the mirror neuron system subserves a wide variety of behaviors including social communication and motor learning (Jeannerod, 1994; Gallese and Goldman, 1998; Rizzolatti et al., 2001; Rizzolatti and Craighero, 2004; Fogassi et al., 2005; Iacoboni et al., 2005). Involvement of the mirror neuron system in motor learning would be supported strongly by evidence demonstrating that observation alone leads to the formation of physiological motor memories akin to those that underlie practiceinduced changes in motor representations. Such evidence has been lacking so far.

In humans, neurophysiological (Fadiga et al., 1995; Hari et al., 1998; Cochin et al., 1999; Brighina et al., 2000; Nishitani and Hari, 2000; Strafella and Paus, 2000; Sundara et al., 2001; Maeda et al., 2002) and neuroimaging (Grafton et al., 1996; Decety et al., 1997; Iacoboni et al., 1999; Grezes and Decety, 2002; Astafiev et al., 2004; Fogassi et al., 2005) studies have revealed mirror activity in several brain regions including the human primary motor cortex (M1). Previously, we developed a paradigm by which the presence of a practice-induced elementary motor memory in the M1 can be demonstrated directly (Classen et al., 1998). After the repetitive performance of unidirectional stereotyped thumb movements, the direction of thumb movements evoked subsequently by transcranial magnetic stimulation (TMS) over the M1 
was changed into the direction of the training, and the excitability of muscle representations in the M1 was changed in favor of the training agonist muscle (Classen et al., 1998, 1999; Bütefisch et al., 2000). Because neurons in this area are active with performance of motor actions and with observation of another individual performing the same motor task, it is conceivable that action observation could form motor memories. The present study tests this hypothesis by examining whether observation of simple finger movements alone can elicit an encoding process in the M1 similar to that induced by practice.

A preliminary account of the results has been published in abstract form (Stefan et al., 2003).

\section{Materials and Methods \\ Subjects}

Twelve right-handed (Oldfield, 1971) subjects (seven men; five women; mean age, $34 \pm 7.6$ years; range, $22-45$ years), naive to the purpose of the experiments, participated in the study. All volunteers had normal physical and neurological examinations and gave written informed consent. This study was approved by the Institutional Review Board of the National Institute of Neurological Disorders and Stroke.

\section{Recording and stimulation procedures}

Subjects sat comfortably in an armchair with the right arm immobilized in a semipronated position in a molded armrest with the four long fingers supported in a slightly extended position and the thumb left entirely free.

Electromyographic recordings (EMGs) were obtained from surface electrodes placed over the belly of the right extensor pollicis brevis (EPB) and right flexor pollicis brevis (FPB) muscles. EMG activity was amplified using a Dantec (Skovlunde, Denmark) Counterpoint electromyograph, bandpass filtered between 10 and $3000 \mathrm{~Hz}$, and fed into a laboratory computer for later off-line analysis. Kinematic measures were obtained with a two-dimensional accelerometer that detected motions in the horizontal (adduction and abduction) and vertical (extension and flexion) axes (Kistler Instrument, Amherst, NY) mounted on the distal phalanx of the thumb. The direction of TMS-evoked and voluntary thumb movements was calculated from the first-peak acceleration vectors in the horizontal and vertical axes in the principal movement plane (Classen et al., 1998). Acceleration signals were digitized at $3000 \mathrm{~Hz}$. Data were analyzed using a data collection-analysis program written in LabView (National Instruments, Austin, TX). TMS was delivered from a custom-built magnetoelectric stimulator (Cadwell Laboratories, Kennewick, WA) through a figure-eight-shaped magnetic coil (wing diameter, $7.0 \mathrm{~cm}$ ) with the subject at rest. The subject's head was kept in an upright position and immobilized by a custom-built aluminum frame. The magnetic coil was connected firmly to the frame so that it remained in a constant position with respect to the head during the experiment. Head and coil stability were monitored with a three-dimensional laser system, as described previously (Bütefisch et al., 2000). Muscle relaxation was monitored by auditory and visual feedback by EMG recording at a sensitivity of $50 \mu \mathrm{V}$ per division. The magnetic coil was placed tangentially to the skull and rotated $45^{\circ}$ away from the midline, approximately perpendicular to the central sulcus, which is the optimal position to activate the corticospinal tract transsynaptically (Werhahn et al., 1994). The optimal scalp position for eliciting isolated thumb movements was identified using a slightly suprathreshold stimulus intensity and marked directly on the scalp with a soft-tip pen. Resting motor threshold (RMT) for the FPB and EPB was defined at this position as the minimum TMS intensity that evoked a motor evoked potential (MEP) of $\geq 50 \mu \mathrm{V}$ in at least 5 of 10 trials in the resting target muscle (Rossini et al., 1994). TMS was then adjusted and applied to this position to elicit isolated and directionally consistent thumb movements.

\section{Experimental design}

Ten subjects participated in three randomly ordered and counterbalanced sessions separated by at least $48 \mathrm{~h}$, which tested the ability of motor practice and observation of another individual performing the practice task on encoding of an elementary motor memory.

\section{Training}

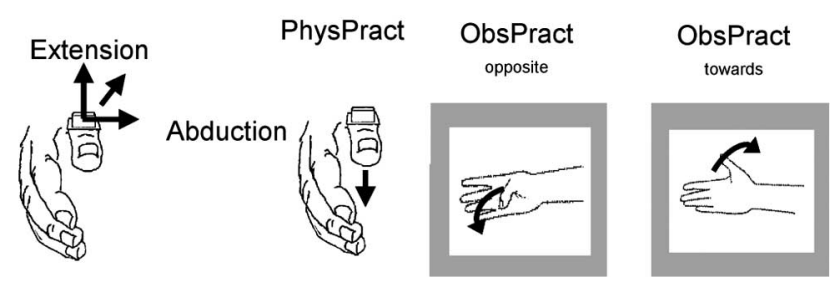

Figure 1. Experimental design. At the beginning of each session, the direction of TMSevoked movements ("baseline before training") was determined in each individual by assessing the first-peak accelerations along the extension/flexion and abduction/adduction axes (Classen et al., 1998). Subsequently, subjects participated in three different training sessions: physical training, which consisted of performance of voluntary thumb movements in a direction opposite the baseline direction and observation of thumb movements directed opposite the baseline direction and toward the baseline direction.

The encoding process was evaluated in each of the three experimental sessions using an established protocol (Classen et al., 1998; Bütefisch et al., 2000). At baseline, 60 TMS stimuli were delivered at $0.1 \mathrm{~Hz}$ to the optimal scalp site to elicit focal thumb movements. These stimuli occasionally elicited perceptions of movement but never allowed the subjects to identify the direction of the motions. After determination of the baseline TMS-evoked thumb movement direction, subjects underwent each of the three interventions for $30 \mathrm{~min}$ in separate sessions. At the end of each intervention, TMS-evoked thumb movement directions were determined again as done for baseline determinations. The principal design of the experiment is shown in Figure 1.

Observational practice (observational practice toward $_{\text {and }}$ abservational practice $_{\text {opposite }}$ ). Subjects were instructed to observe attentively brisk repetitive thumb movements displayed at their natural size on a computer screen $1 \mathrm{~m}$ in front of them. With their gaze directed straight ahead during the video presentation (see below), the subject's hand was outside their field of view. Observed motions were performed by a white male actor, cued at $1 \mathrm{~Hz}$ in either an extension or flexion direction (two different videos were used according to the TMS-evoked thumb movement directions determined at baseline). The actor's right hand was viewed from a frontal plane, with surface electrodes and accelerometer positioned as during physical practice (see below). Because most TMSevoked thumb movements were predominantly oriented along an extension/flexion axis, the video movements were approximately collinear to this principal direction. In the video displaying extension movements, a total of $6 \%$ (108 of a total of 1800) of all movements were directed opposite to the other $94 \%$ (i.e., in a flexion direction). These movements were interspersed randomly in between the extension movements. Similarly, in the video showing flexion movements, $6 \%$ of all movements were in the opposite direction. Total duration of the videos was $30 \mathrm{~min}$ each. Subjects were instructed to remain relaxed throughout the observation of the videos. Absence of voluntary activity was monitored by EMG recordings at a sensitivity of $50 \mu \mathrm{V}$ per division. If voluntary EMG activity was detected, subjects were instructed to relax. EMG activity during physical or observational practice was quantified in each subject by computing the mean area under the curve for EMG activity of the training agonist in $\sim 70$ epochs of $200 \mathrm{~ms}$ duration sampled randomly throughout the training. In physical practice, EMG activity only related to the training motions was computed. In observational practice, EMG activity was integrated over the entire length of $200 \mathrm{~ms}$ epochs sampled during the observations. Therefore, the area under the curve in observational practice is a conservative measure of unwanted EMG activity during these conditions.

Observational practice was implemented in two different sessions. For observational practice toward $_{\text {(ObsPract }}$ toward $)$, subjects were shown the video displaying thumb movements in the same principal direction as the baseline direction, as determined during the 60 magnetic stimulations beforehand. For example, if the principal direction of TMS-evoked thumb movements was extensor, then thumb extension movements 
Table 1. Behavioral characteristics

\begin{tabular}{|c|c|c|c|c|}
\hline Training condition & $\begin{array}{l}\text { Alertness } \\
\text { (arbitrary units, } \\
\text { visual analog scale) }\end{array}$ & $\begin{array}{l}\text { Attention } \\
\text { (arbitrary units, } \\
\text { visual analog scale) }\end{array}$ & $\begin{array}{l}\text { Number of missed } \\
\text { directionally deviant movements } \\
\text { (of 108) }\end{array}$ & $\begin{array}{l}\text { Area under the curve of voluntary } \\
\text { (PhysPract) or involuntary (ObsPract) } \\
\text { EMG activity (training agonist, } \mathrm{mV} \cdot \mathrm{ms} \text { ) }\end{array}$ \\
\hline PhysPract & $5.6 \pm 1.0$ & $6.9 \pm 0.8$ & n.a. & $134.0 \pm 26.8$ \\
\hline ObsPract $_{\text {opposite }}$ & $6.6 \pm 0.8$ & $6.6 \pm 0.6$ & $6.0 \pm 2.3$ & $0.9 \pm 0.1$ \\
\hline ObsPract towards & $5.5 \pm 1.2$ & $7.9 \pm 0.8$ & $4.2 \pm 0.8$ & $0.9 \pm 0.2$ \\
\hline
\end{tabular}

n.a., Not applicable.

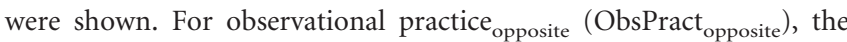
video showed movements opposite to the baseline direction. For example, if the principal baseline direction was extension, then the video showing flexor movements was presented to the subject. To maintain similar attention and motivation during the $30 \mathrm{~min}$ of the video presentation, subjects were instructed to count silently the number of interspersed directionally deviant thumb movements $(6 \%)$. Error rate was defined as the absolute difference of the number of interspersed deviant movements as identified by the subject and the correct number. Subjects were instructed that they would be financially rewarded according to their performance in the counting task. At the end of each session, subjective assessments of alertness during the experiments and attention to the observation were scored by the subjects using a visual analog scale ranging from -10 to +10 . Additionally, the subjects were asked the question, "Did you imagine yourself performing the thumb movements you have watched?" to determine whether or not they had experienced imagery during the video presentations.

Physical practice. Subjects were instructed to perform voluntary brisk thumb movements visually paced by a light-emitting diode blinking at 1 $\mathrm{Hz}$ in a direction approximately opposite to the baseline direction (Classen et al., 1998; Bütefisch et al., 2000). For example, if the principal baseline direction was extension, then the subjects were instructed to perform repetitive flexion movements. Thus, physical practice (Phys-

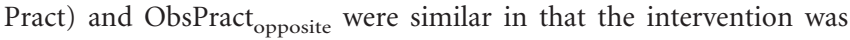
directed away from the baseline of TMS-evoked movements determined beforehand. After each single training movement, the thumb returned to the original position by relaxation. The total duration of practice was 30 min. Practiced thumb movements were monitored closely on-line with respect to directional consistency of training movements by the experimenter. In addition to the TMS-evoked movements, all practiced movements were recorded during training for later off-line analysis.

All interventions were each organized in three blocks of $10 \mathrm{~min}$, separated by $2.5 \mathrm{~min}$ of rest. In between any two blocks, 12 thumb movements were evoked by delivering TMS at a frequency of $0.1 \mathrm{~Hz}$. Acceleration and EMG signals related to TMS-evoked movements were recorded as described above.

Four subjects participated in additional experiments to determine the influence of observational practice displaying (1) $50 \%$ thumb movements in each of the two different directions (flexion-extension; ObsPract ${ }_{50 / 50}$ ) and (2) focusing attention on the frequently $(94 \%)$ or infrequently (6\%) observed motions (ObsPract opposite $^{94 \%}$ and ObsPract ${ }_{\text {opposite }} \%$, respectively).

\section{Data analysis}

Encoding of a motor memory: movement direction. Directions of TMSevoked movements and of voluntary movements were derived from the first-peak accelerations in the two orthogonal axes in the principal movement plane (Classen et al., 1998) determined independently by two investigators, one of them blind to the intervention. The baseline direction of thumb movements was defined as the direction of the mean vector of all vectors recorded during baseline. To describe intervention effects on TMS-evoked thumb movement directions, we defined a training target zone (TTZ) as a window of $\pm 20^{\circ}$ centered on the direction of the performed (PhysPract) (Bütefisch et al., 2000) and observed (ObsPract) training motions. In the PhysPract session, the target direction was determined as the direction of the mean vector of 360 vectors representing every fifth movement performed during the training. In the ObsPract (ObsPract $_{\text {opposite }}$ and ObsPract toward ) sessions, the target direction was either vertical up $\left(90^{\circ}\right)$ or vertical down $\left(270^{\circ}\right)$, corresponding to the direction of the thumb movements displayed to the subject. The measure for direction was the probability of a TMS-evoked thumb movement to fall into the TTZ, a measure consistent, reproducible, and capable of identifying training-dependent changes in the organization of the M1 (Bütefisch et al., 2000). This probability was estimated by the percentage of TMS-evoked thumb movements falling into the TTZ $\left(P_{\mathrm{TTZ}}\right)$ relative to the number of all TMS-evoked thumb movements, according to the following equation (Bütefisch et al., 2000): $P_{\mathrm{TTZ}}=N_{\mathrm{TTZ}} / N_{\text {total }} \times 100$, where $N_{\text {TTZ }}$ is the number of all TMS-evoked thumb movements falling into the TTZ, and $N_{\text {total }}$ is the number of all TMS-evoked thumb movements elicited within a given time period.

Intervention effects across conditions were compared by computing $\Delta P_{\mathrm{TTZ}}$ according to the following equation: $\Delta P_{\mathrm{TTZ}}=P_{\mathrm{TTZ} \_ \text {after }}-$ $P_{\text {TTZ_before. }}$.

Compound acceleration vector. The effect of training was also determined by comparing the mean magnitude of the first-peak acceleration in the extension/flexion direction before and after training. Intervention effects on the magnitude of first-peak acceleration of TMS-evoked thumb movements were calculated by normalizing all baseline vectors to the same hemispheric direction (extension).

Corticomuscular excitability. MEP amplitudes were measured peak to peak in each individual trial. $\mathrm{MEP}_{\text {agonist }}$ and $\mathrm{MEP}_{\text {antagonist }}$ refer to MEPs recorded from muscles acting either as agonist or antagonist of the trained (either by physical practice or by observation) movement direction in each subject. For example, with practice or observation of extension movements, the EPB was designated as the agonist muscle, and the FPB was designated as the antagonist muscle. $\mathrm{MEP}_{\text {agonist }}$ and $\mathrm{MEP}_{\text {antagonist }}$ were each normalized to baseline. To describe the net effects of training on corticomuscular excitability, the ratio $E$ between $\mathrm{MEP}_{\text {agonist }}$ and $\mathrm{MEP}_{\text {antagonist }}$ was determined both before and after training, according to the following equation: $E_{\text {ratio }}=\mathrm{MEP}_{\text {agonist }}$ I MEP antagonist .

Unless stated otherwise, intervention effects on movement kinematics and corticomuscular excitability were analyzed by repeated-measures ANOVA (ANOVA $\left.A_{\mathrm{RM}}\right)$. Two-tailed paired $t$ tests were applied for exploratory and post hoc analyses, as appropriate. The false discovery rate procedure was used for correction for multiple comparisons (CurranEverett, 2000).

Data were expressed as mean \pm SEM. Effects were considered significant if $p<0.05$.

\section{Results}

Subjects' rating of alertness $\left(\mathrm{ANOVA}_{\mathrm{RM}} ; F=0.9 ; \mathrm{NS}\right)$ and attention to the task $\left(\mathrm{ANOVA}_{\mathrm{RM}} ; F=1.2\right.$; NS) did not differ across training conditions (Table 1). Error rates in detection of deviant movements during observation were similar in ObsPract $_{\text {opposite }}$ and ObsPract toward (NS; paired $t$ test). Background EMG activity, minimal during observational practice, was comparable between ObsPract $_{\text {opposite }}$ and ObsPract ${ }_{\text {toward }}$ and also comparable between agonist and antagonist muscles (Table 1). All subjects denied imagining themselves performing the observed movements.

\section{Effects on movement direction}

Determination of movement direction by the two investigators were highly consistent $(r=0.985 ; p<0.05)$. Training direction differed from the baseline direction by $152.7 \pm 5.2^{\circ}$ in PhysPract, 
A

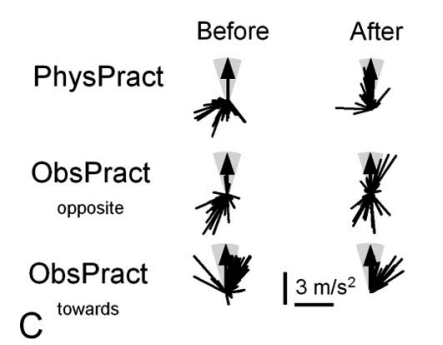

B

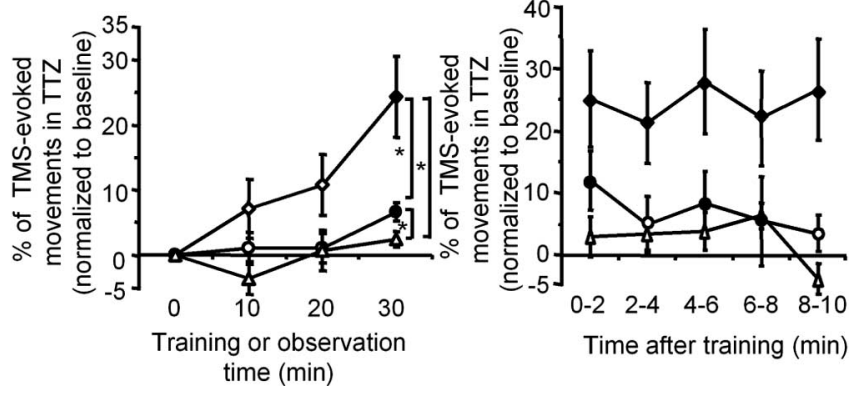

Figure 2. Effect of different training interventions on TMS-evoked movement direction. $\boldsymbol{A}$, Example of one subject. First-peak acceleration vectors of TMS-evoked thumb movements before (left) and after (right) each of the three interventions (PhysPract, ObsPract ${ }_{\text {opposite, }}$ and ObsPract toward $_{\text {ard }}$. For better comparability, all examples are aligned to the training direction indicated by the arrow. The TTZ is shown in gray. PhysPract and ObsPract ${ }_{\text {opposite }}$ but not ObsPract toward led to substantial changes in the direction of TMS-evoked movements. $\boldsymbol{B}$, Group data $(n=10)$ showing the $P_{T T Z}$ before and after PhysPract, 0 bsPract ${ }_{\text {opposite, }}$ and 0 bsPract ${ }_{\text {toward }}$. Before the training interventions, the percentage of TMS-evoked movements in TTZ was similar in PhysPract and ObsPract ${ }_{\text {opposite. }}$ PhysPract and ObsPract ${ }_{\text {opposite, }}$ but not ObsPract toward led to a significant increase in the percentage of TMS-evoked movements falling into TTZ. Data show means \pm SEM. ${ }^{*} p<0.005$. n.S., Not significant. $C$, Time course of changes of $P_{\mathrm{TTZ}}$ as a function of training intervention. To compare between conditions, $\Delta P_{\mathrm{TTZ}}$ was computed as the baselinenormalized, intervention-dependent change in percentage of TMS-evoked thumb movements falling into the TTZ (see Materials and Methods). At the end of $30 \mathrm{~min}$, the percentage change of TMS-evoked movements in TTZ was larger in PhysPract than in ObsPract ${ }_{\text {opposite }}$ or ObsPract $_{\text {toward }}$ and with ObsPract opposite $_{\text {than with ObsPract }}$ toward. Rhombi, PhysPract; circles, ObsPract ${ }_{\text {opposite; }}$ triangles, ObsPract toward . Filled symbols, Time points significantly different from baseline; two-tailed $t$ tests; false discovery rate correction. ${ }^{*} p<0.05$. $\boldsymbol{D}$, Duration of changes of $P_{\mathrm{TTZ}}$ as a function of training intervention. Symbols as in $C$. Error bars represent SEM.

by $152.6 \pm 7.4^{\circ}$ in ObsPract $_{\text {opposite, }}$, and by $26.1 \pm 5.3^{\circ}$ in ObsPract ${ }_{\text {toward }}$.

PhysPract and ObsPract ${ }_{\text {opposite }}$ resulted in an increase in $P_{\text {TTZ }}$ (see Materials and Methods), more prominent with the former than with the latter, in the absence of changes with ObsPract ${ }_{\text {toward }}$. An example of the findings is shown in Figure 2A.

Group data showed significant effects of time (before, after) $\left(\right.$ ANOVA $\left._{\mathrm{RM}} ; F=20.7 ; p<0.005\right)$, training type (PhysPract, ObsPract $_{\text {opposite }}$, ObsPract toward $\left._{\text {ow }}\right)(F=3.6 ; p=0.05)$, and of the interaction term time-by-training type $(F=11.0 ; p<0.005)$ on $P_{\text {TTZ }}$ (Fig. 2B). $P_{\text {TTZ }}$ increased significantly with PhysPract (from $2.4 \pm 1.0$ to $26.6 \pm 6.9 \% ; p<0.005$ ) and with ObsPract $_{\text {opposite }}$ (from $2.6 \pm 0.8$ to $9.1 \pm 1.5 \% ; p<0.005$ ) but not

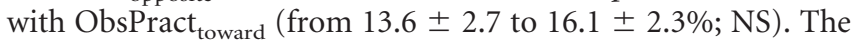
increase in $P_{\text {TTZ }}$ with ObsPract opposite $_{\text {was }}$ evident in every single individual tested.

The time course of the build-up and decay, respectively, of training-dependent directional changes was assessed separately for each training type. PhysPract required no less training blocks than ObsPract $_{\text {opposite }}$ to result in a significant increase of $P_{\text {TTZ }}$ [three blocks (i.e., $30 \mathrm{~min}$ ); two-tailed $t$ tests; false discovery rate correction]. However, the magnitude of the effect was different between the two effective training types. After $30 \mathrm{~min}, \Delta P_{\mathrm{TTZ}}$, the baseline-normalized percentage of TMS-evoked movements falling within the TTZ (see Materials and Methods), was larger in PhysPract than in ObsPract ${ }_{\text {opposite }}(p<0.02$; two-tailed $t$ test) and ObsPract toward $(p<0.01)$ and larger in ObsPract $_{\text {opposite }}$ than in ObsPract toward $_{\text {( }}(p<0.05$; two-tailed $t$ test; false discovery rate correction) (Fig. 2C). To analyze the duration of observationinduced changes of $P_{\text {TTZ}}$, the data were binned in epochs of $2 \mathrm{~min}$ for a total of $10 \mathrm{~min}$ after the end of training. For each training type, each epoch was compared with baseline using two-tailed $t$ tests. After false discovery rate correction, $P_{\text {TTZ }}$ was significantly different from baseline for PhysPract in all five bins $(0-10 \mathrm{~min})$ and for ObsPract ${ }_{\text {opposite }}$ in the first four bins, except epoch $2(0-8$ $\mathrm{min}$ ), in the absence of changes with ObsPract ${ }_{\text {toward }}$ in any of the five bins (Fig. 2D). These findings suggested that the effect induced by ObsPract ${ }_{\text {opposite }}$ was less pronounced and, possibly, shorter lasting that that induce by PhysPract.

To examine the possibility that the increase of $P_{\mathrm{TTZ}}$ in ObsPract $_{\text {opposite }}$ was unrelated to the direction of the observed thumb movements, in four subjects, ObsPract ${ }_{\text {opposite }}$ was compared with observation of a video displaying an equal number of flexion and extension thumb movements $\left(\mathrm{ObsPract}_{50 / 50}\right)$. In this session, a ${ }^{*} \mathrm{TTZ}^{\star}$, directed oppositely to the baseline direction, was operationally defined in analogy to the TTZ in the ObsPract $_{\text {opposite }}$ condition (as outlined above). Observing directionally balanced thumb movements did not increase $P_{*_{\mathrm{TTZ}}}$. Importantly, in the same subjects, two additional experiments demonstrated that ObsPract ${ }_{\text {opposite }}$ effectively increased $P_{\text {TTZ. In }}$ these experiments, ObsPract ${ }_{\text {opposite }}$ elicited an increase in the percentage of TMS-evoked movements falling into the TTZ regardless of whether the frequent target movements or the infrequent directionally deviant movements were counted by the subject. $\Delta P_{\text {TTZ }}$ did not differ statistically significantly between the two ObsPract conditions (ObsPract ${ }_{\text {opposite }} 96 \%: \Delta P_{\text {TTZ }}, 4.5 \pm 2.0 \%$; ObsPract $_{\text {opposite } 6 \%}: \Delta P_{\text {TTZ }}, 8.9 \pm 2.6 \%$ ) but was different between any of the ObsPract ${ }_{\text {opposite }}$ conditions and ObsPract $_{50 / 50}$ $\left(\Delta P_{{ }^{*} \mathrm{TTZ}^{*}},-0.5 \pm 1.3 \%\right.$; NS $)$.

\section{Effects on compound acceleration vector}

We considered the net first-peak acceleration along the extension/flexion axis of the entire population of acceleration vectors of the TMS-evoked thumb movements. This was termed the compound acceleration vector. The compound acceleration vector is sensitive to the magnitude of acceleration and to changes in the acceleration of movements falling outside of the TTZ and, thus, complements the analysis of the percentage of movements falling within TTZ. At baseline, the mean absolute magnitude of first-peak acceleration was comparable across experimental conditions (Table 2).

Practicing thumb movements in the training direction caused the mean first-peak acceleration vector to change into the training direction, in agreement with previous findings (Classen et al., 1998). With ObsPract ${ }_{\text {opposite, }}$, the compound acceleration vector length decreased, whereas it did not change appreciably with

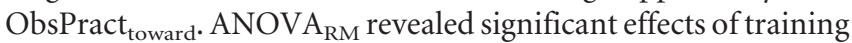
type (PhysPract, ObsPract opposite, $_{\text {ObsPract }}$ toward $)(F=8.4 ; p<$ $0.01)$, time (before, after) $(F=15.3 ; p<0.01)$, and of the interaction training type-by-time $(F=10.0 ; p<0.01)$ (Fig. $3 A)$.

With PhysPract, the first-peak acceleration changed from $0.5 \pm 0.1$ to $-0.3 \pm 0.2 \mathrm{~m} / \mathrm{s}^{2}(p<0.01$; paired two-tailed $t$ test $)$, with ObsPract ${ }_{\text {opposite }}$ from $0.4 \pm 0.0$ to $0.1 \pm 0.1 \mathrm{~m} / \mathrm{s}^{2}(p<0.01)$, and with ObsPract toward from $0.6 \pm 0.1$ to $0.6 \pm 0.1 \mathrm{~m} / \mathrm{s}^{2}$ (NS). This finding suggests that with ObsPract $_{\text {opposite, the total }}$ acceleration along the axis of the observed movements had 
Table 2. Baseline measures in each of the three training sessions

\begin{tabular}{lllll}
\hline Training condition & $\begin{array}{l}\text { RMT MEP } \\
\text { agonist }\end{array}$ & $\begin{array}{l}\text { TMS stimulus } \\
\text { intensity (\%) }\end{array}$ & $\begin{array}{l}\text { Peak acceleration of TMS- } \\
\text { evoked movements (m/s } s^{2}\end{array}$ & $\begin{array}{l}\text { MEP } \\
\text { agonist } \\
\text { amplitude (mV) }\end{array}$ \\
\hline PhysPract & $58 \pm 3$ & $68 \pm 2$ & $0.5 \pm 0.3$ & $1.4 \pm 0.2$ \\
ObsPract $_{\text {opposite }}$ & $58 \pm 2$ & $68 \pm 2$ & $0.4 \pm 0.1$ & $1.2 \pm 0.2$ \\
ObsPract $_{\text {towards }}$ & $58 \pm 2$ & $67 \pm 2$ & $0.5 \pm 0.3$ & $1.7 \pm 0.3$ \\
\hline
\end{tabular}

${ }^{a}$ TMS stimulus intensity required to elicit isolated thumb movements at baseline (percentage of maximal stimulator output).

A

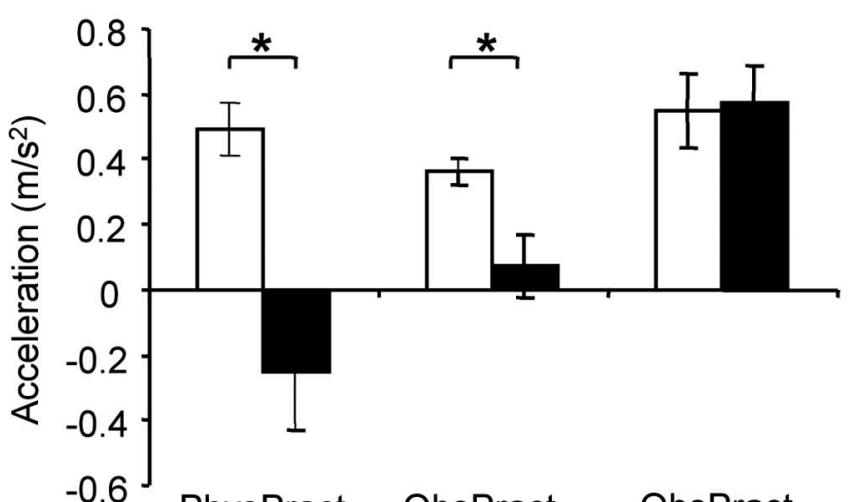

B

PhysPract ObsPract ObsPract

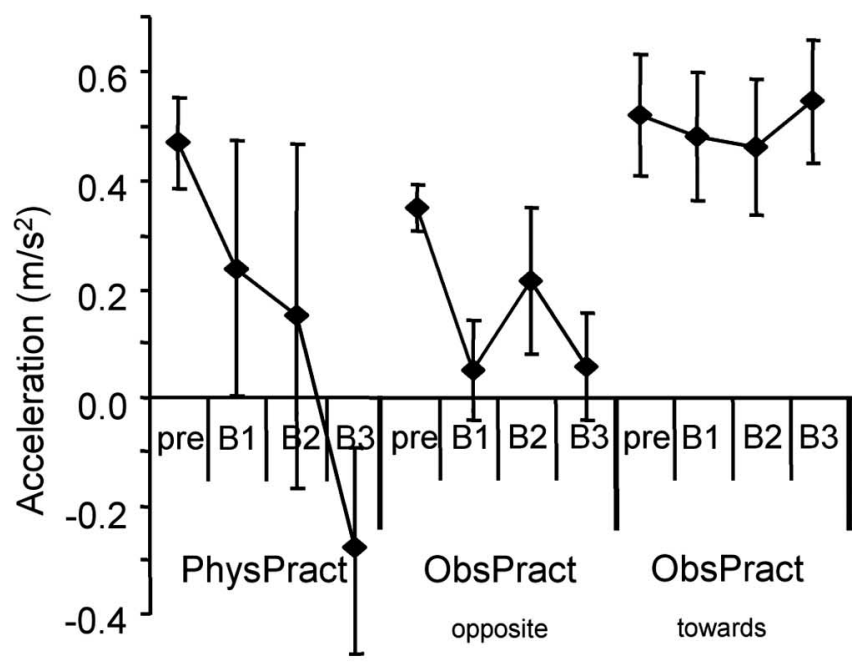

Practice or observation time (min)

Figure 3. Effect of different training interventions on compound acceleration vector. $A$, Group data $(n=10)$ showing the mean compound acceleration vector for the extension/flexion direction before and after training. Before training, the compound acceleration vector of each subject was aligned such that all vectors pointed into the extension direction. After PhysPract, the mean compound acceleration vector came to point into the flexion direction, corresponding to the practiced direction. ObsPract ${ }_{\text {opposite }}$ decreased the length of the mean compound acceleration vector, whereas $0 \mathrm{bsPract}{ }_{\text {toward }}$ did not change the mean compound acceleration vector. ${ }^{*} p<0.01$. Open bars, Before training; filled bars, after training. $\boldsymbol{B}$, Time course of changes of the mean compound acceleration vector as a function of training intervention. At the end of 30 $\mathrm{min}$, the mean compound acceleration vector was smaller in PhysPract than in 0 bsPract ${ }_{\text {opposite }}$ or ObsPract ${ }_{\text {toward }}$ and with 0 bsPract ${ }_{\text {opposite }}$ than with ObsPract toward $_{\text {. }}$. Error bars represent SEM. pre, Before training; $B 1, B 2$, and $B 3$, results obtained after the first, second, and third training block, respectively.

changed in favor of movements directed away from the baseline direction.

The time course of the build-up of training-dependent changes of first-peak acceleration was assessed separately for each

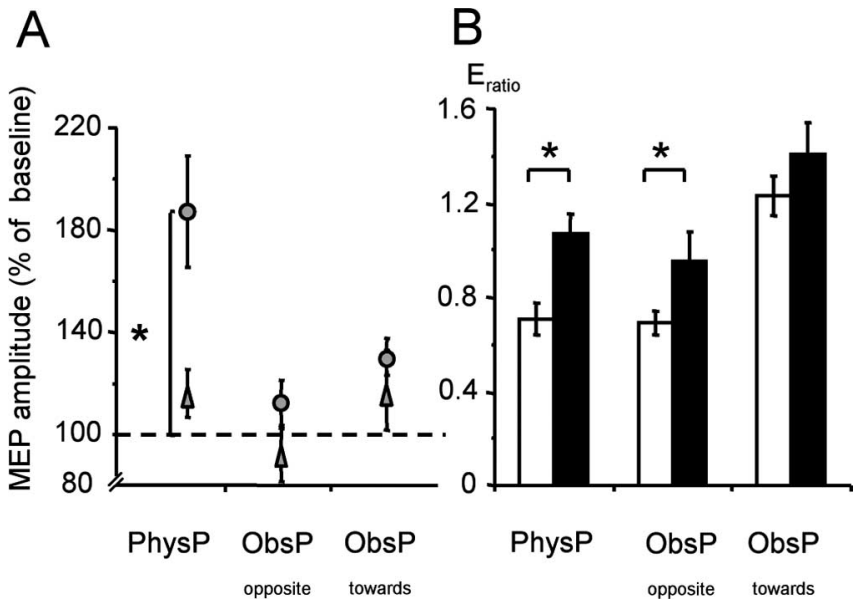

Figure 4. Effect of different training interventions on corticomuscular excitability. $\boldsymbol{A}$, Changes in MEP amplitudes (relative to baseline) recorded from training agonist muscle (MEP agonist; $i$ ircles) and antagonist muscle ( $M E P_{\text {antagonist }}$ triangles). PhysPract led to a significant increase in $M P_{\text {agonist }}$ compared with baseline. $\boldsymbol{B}$, The ratio between the $M E P_{\text {agonist }}$ and MEP $_{\text {antagonist }}$ increased both after PhysPract and ObsPract ${ }_{\text {opposite }}$ but not after ObsPract ${ }_{\text {toward }}$. Open bars, Before training; filled bars, after training. ${ }^{*} p<0.05$. Error bars represent SEM.

training type. PhysPract required no less training blocks than ObsPract $_{\text {opposite }}$ to result in a significant decrease of first-peak acceleration [three blocks (i.e., $30 \mathrm{~min}$ ); two-tailed $t$ tests; false discovery rate correction]. In fact, first-peak acceleration differed

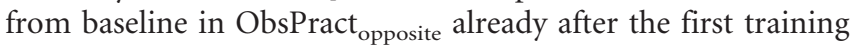
block. After $30 \mathrm{~min}$ of training, first-peak acceleration was smaller (indicating a larger training effect) in PhysPract than that in ObsPract toward $(p<0.005$; two-tailed paired $t$ test $)$ and in ObsPract $_{\text {opposite }}$ than that in ObsPract toward $(p<0.005)$ (Fig. 3B), whereas the difference between PhysPract and ObsPract ${ }_{\text {opposite }}$ was not statistically significant. Similar results emerged when only movements outside the TTZ were considered (data not shown).

\section{Effects on corticomuscular excitability}

The size of the TMS-evoked motor potentials in the EPB and FPB was determined. MEP size was stratified according to the role of the muscle in the training. At baseline, measures of corticomuscular excitability (RMT, MEP amplitudes) were comparable across experimental conditions (Table 2).

ANOVA $_{\mathrm{RM}}$ revealed significant effects of training type (PhysPract, ObsPract opposite $_{\text {, ObsPract }}$ toward $)(F=4.7 ; p<0.05)$ and of training action (agonist, antagonist) $(F=37.0 ; p<0.05)$. There was a strong statistical trend for the interaction term training action-by-training type $(F=3.5 ; p=0.051)$ (Fig. $4 A)$.

With PhysPract, $\mathrm{MEP}_{\text {agonist }}$ increased, on average, by $82 \%$ ( $p<0.02$; two-tailed paired $t$ test), in agreement with previous findings (Classen et al., 1999; Bütefisch et al., 2000), whereas $\mathrm{MEP}_{\text {antagonist }}$ remained unchanged. No significant changes of MEP amplitudes were noted in either the training agonist or training antagonist in any of the observational training 
conditions. RMT as measured in five subjects before and after ObsPract $_{\text {opposite }}$ remained unchanged (RMT before, $52 \pm 3 \%$ of maximal stimulator output; RMT after, $53 \pm 3$ of maximal stimulator output; NS). Movements may be more adequately described by the balance of corticomuscular excitability rather than by the magnitude of excitability of either the training agonist or antagonist. Therefore, the effect of training was assessed by comparing the ratio between $\mathrm{MEP}_{\text {agonist }}$ and $\mathrm{MEP}_{\text {antagonist }}\left(E_{\text {ratio }}\right)$ before and after training, separately for each training intervention. Two-tailed $t$ tests revealed that this ratio increased with PhysPract $(p<0.01)$ and also with ObsPract opposite $(p<0.05)$, whereas it

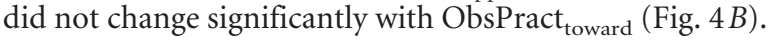

\section{Discussion}

The present study has shown that mere observation of movements led to the formation of a lasting specific memory trace in movement representations that resembled that elicited by physical training.

\section{Observation-induced motor memory}

After observation of movements directed away from the baseline movement direction, the probability of TMS-evoked movements to fall into the training target zone increased, the net acceleration of all TMS-evoked thumb movements was changed toward the direction of the observed movements, and the balance of corticomuscular excitability was altered in favor of the agonist of the observed movements. Changes of motor measures were not noted when the ObsPract movements were oriented toward the baseline direction or when the direction of the observed movements on balance was undetermined. Thus, although observation-induced changes in motor representation were substantially smaller compared with those induced by physical practice, they were highly similar with respect to both quality and specificity. It seems unlikely that observation-induced plasticity of motor representations in the present study was merely a consequence of involuntary movements during movement observation, because involuntary EMG activity during observation was found to be smaller by several orders of magnitude than that associated with physical training (Table 1). Therefore, our findings provide evidence for the notion that observation alone may induce lasting specific changes in motor representation, a kind of motor memory.

Evidence derived from studies involving the physical practice of movements suggests that formation of a motor memory may be an important physiological step in motor skill acquisition (Karni et al., 1995; Pascual-Leone et al., 1995; Kleim et al., 1998; Sanes and Donoghue, 2000; Conner et al., 2003). Therefore, our data raise the intriguing possibility that observational practice may improve motor performance (Black and Wright, 2000; Heyes and Foster, 2002; Horn et al., 2002; Edwards et al., 2003; Petrosini et al., 2003) by mechanisms similar to those involved in motor skill acquisition by physical training. Our findings are consistent with results demonstrating that motor performance may be facilitated even with observation of simple movements (Brass et al., 2001) and with observational motor tasks that are devoid of explicit cognitive components (Vogt, 1995; Vinter and Perruchet, 2002; Mattar and Gribble, 2005).

\section{Mechanism and location of observation-induced plasticity}

As mentioned above, the ventral premotor cortex (vPMC) contains mirror neurons that fire both with action observation and action execution (Gallese et al., 1996; Rizzolatti et al., 1996). The ventral PMC contains representations of distal upper extremity movements (Fogassi et al., 2001). Just as canonical neurons (Kakei et al., 1999, 2001), mirror neurons (Gallese et al., 1996; Fadiga et al., 2000) in the vPMC may encode information on the direction of voluntary movements in an extrinsic coordinate system. Although mirror neurons in the vPMC mostly respond to the perception of specific hand-object interactions or goals (Gallese et al., 1996; Gallese and Goldman, 1998; Rizzolatti et al., 2001), activation of the PMC has also been observed with intransitive movements similar to the movements tested in the present study (Iacoboni et al., 1999). Furthermore, because some mirror neurons do not discharge with observation of a single grasping action but only after repeated presentation of such action (Rizzolatti and Fadiga, 1998), it is possible that learning, as demonstrated previously in canonical premotor neurons (Mitz et al., 1991), extends to mirror neurons. These physiological properties may suggest involvement of vPMC neurons in observationinduced plasticity. It has been shown that M1 neurons are facilitated at short latencies after PMC activation via dense premotor-M1 connections (Cerri et al., 2003; Shimazu et al., 2004). Therefore, mirror activity could have been induced in the M1 by transmission of mirror neuron activity from the PMC. Alternatively, it is conceivable that the M1 may harbor genuine mirror neurons. Although mirror neurons, responsive to both movement observation and execution, have not been found in direct recordings in nonhuman primates (Gallese et al., 1996), indirect evidence for their presence in human M1 may be inferred from various studies in humans (Fadiga et al., 1995; Hari et al., 1998; Grezes and Decety, 2002). Independent of its anatomic identity, mirror activity in the M1, whether arising as a consequence of activity in local genuine mirror neurons in the M1 or of transmission from the vPMC onto the M1, has been demonstrated to preserve the temporal structure of observed movements at high fidelity (Nishitani and Hari, 2000, 2002; Gangitano et al., 2001). Therefore, local neuronal activity in the M1 appears to be equipped with necessary properties to encode a local memory that retains details of the observed movements in all relevant aspects.

Conceivably, specific patterns of neuronal activations during motor observation could have been transmitted to the spinal cord (e.g., through observation-induced activation of direct spinal connections from the vPC or M1). This could have led to lasting local specific changes of spinal excitability. Spinal excitability changes during action observation have indeed been reported in one study (Baldissera et al., 2001) but have been absent in another study (Patuzzo et al., 2003). Even if we cannot rule out that spinal cord excitability may be modifiable during observation, it appears highly unlikely that it could underlie the present specific observation-induced plasticity, because spinal excitability changes during movement observation (facilitation of the movement antagonist) were directed oppositely to the effects found in the present study (Baldissera et al., 2001).

\section{Implications for involvement of the mirror neuron system in motor learning}

The mirror neuron system has been shown to be involved in imitation of familiar elementary movements (Iacoboni et al., 1999). More recently, Buccino et al. (2004) provided evidence that the mirror neuron system was active also when subjects attempted to imitate novel hand postures. Because the hand postures were unfamiliar to the subjects, it was concluded that the mirror neuron system has a role in the acquisition of new motor skills. The authors advanced the view that in this situation, the familiar elements of the observed novel complex movements 
would generate resonant activity within the mirror neuron system. By virtue of this mechanism, the selection of appropriate neuronal activation patterns underlying the intended movement would be greatly facilitated, and the recombination of elementary overlearned movements into new movements of greater complexity could be accomplished more easily (Buccino et al., 2004). Importantly, in imitation-based motor learning, the final necessary brain signal leading to the formation of lasting motor memories was thought to be provided through the physical practice of movements. According to this view, in imitation-based motor learning the mirror neuron system facilitates motor learning through facilitating the physical performance of the appropriate training movements.

Although the study by Buccino et al. (2004) showed that the mirror neuron system is active in imitation of novel movements, indirect neurophysiological evidence for the actual formation of a memory trace was probably first provided by Järveläinen et al. (2004). Mattar and Gribble (2005) used kinematic analyses to show that the acquisition of complex motor behaviors (learning to reach in a novel mechanical environment) is facilitated by previous observation of subjects learning the novel task. Motor learning by observation was impaired when the motor system was engaged with an unrelated movement task. These findings support the view that motor systems are involved in acquiring neural representations of novel environments during observation.

Our results demonstrate that a specific motor memory is formed by observation, one that is similar to that induced by practicing movements. Therefore, the present results provide possibly the most direct evidence to date suggesting that the mirror neuron system may be involved in generating lasting mnemonic representations of movements, a likely prerequisite of motor learning.

Our data also imply that a specific plasticity of movement representation, qualitatively indistinguishable from practiceinduced plasticity, may be generated in the absence of peripheral somatosensory afferent information. How can an observationinduced movement memory be reconciled with the documented critical role of somatosensory afferents (Rothwell et al., 1982) and the somatosensory cortex (Pavlides et al., 1993) in motor cortical plasticity and motor learning? Importantly, movement observation has been shown to modulate activity in human somatosensory regions (Avikainen et al., 2002), and it is possible that this activity supplies the necessary somatosensory signal to the M1 that appears to be a requisite for aspects of motor memory formation.

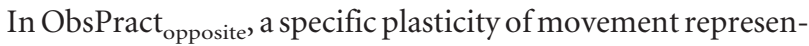
tation was induced independent of whether the subjects counted the frequent target movements or the infrequent directionally deviant movements. Presumably, the conscious cognitive operations involved in deciding whether an observed movement belonged to the target movements differed between these two ObsPract $_{\text {opposite }}$ conditions. Had this been important to the processes involved in generation of motor memories, this likely would have influenced the training results. The finding that the effects of ObsPract ${ }_{\text {opposite }}$ were in fact quite similar when counting the frequent or infrequent movements suggests that conscious cognitive operations were unlikely to be deeply involved in the formation of the new mnemonic representations. This view is further supported by the study by Mattar and Gribble (2005) demonstrating that the beneficial effect of previous observation was uncompromised even when attentional systems were engaged during observation of a distractor task. These findings are additional indirect support for a causal role of the mirror neuron system, which appears to be able to operate primarily independently of conscious cognition.

The present findings suggest that movement observation may have a potential as a technique in rehabilitative settings. Observation-based approaches to aid in the recovery from brain injury may be useful in patients who have difficulties in generating physical movements or who are unable to understand verbal instructions.

\section{References}

Astafiev SV, Stanley CM, Shulman GL, Corbetta M (2004) Extrastriate body area in human occipital cortex responds to the performance of motor actions. Nat Neurosci 7:542-548.

Avikainen S, Forss N, Hari R (2002) Modulated activation of the human SI and SII cortices during observation of hand actions. NeuroImage 15:640-646.

Baldissera F, Cavallari P, Craighero L, Fadiga L (2001) Modulation of spinal excitability during observation of hand actions in humans. Eur J Neurosci 13:190-194.

Black CB, Wright DL (2000) Can observational practice facilitate error recognition and movement production? Res Q Exerc Sport 71:331-339.

Brass M, Bekkering H, Prinz W (2001) Movement observation affects movement execution in a simple response task. Acta Psychol (Amst) 106:3-22.

Brighina F, La Bua V, Oliveri M, Piazza A, Fierro B (2000) Magnetic stimulation study during observation of motor tasks. J Neurol Sci 174:122-126.

Buccino G, Vogt S, Ritzl A, Fink GR, Zilles K, Freund HJ, Rizzolatti G (2004) Neural circuits underlying imitation learning of hand actions; an eventrelated FMRI study. Neuron 42:323-334.

Bütefisch CM, Davis BC, Wise SP, Sawaki L, Kopylev L, Classen J, Cohen LG (2000) Mechanisms of use-dependent plasticity in the human motor cortex. Proc Natl Acad Sci USA 97:3661-3665.

Cerri G, Shimazu H, Maier MA, Lemon RN (2003) Facilitation from ventral premotor cortex of primary motor cortex outputs to macaque hand muscles. J Neurophysiol 90:832-842.

Classen J, Liepert A, Wise SP, Hallett M, Cohen LG (1998) Rapid plasticity of human cortical movement representation induced by practice. J Neurophysiol 79:1117-1123.

Classen J, Liepert J, Hallett M, Cohen L (1999) Plasticity of movement representation in the human motor cortex. Electroencephalogr Clin Neurophysiol Suppl 51:162-173.

Cochin S, Barthelemy C, Roux S, Martineau J (1999) Observation and execution of movement: similarities demonstrated by quantified electroencephalography. Eur J Neurosci 11:1839-1842.

Conner JM, Culberson A, Packowski C, Chiba AA, Tuszynski MH (2003) Lesions of the basal forebrain cholinergic system impair task acquisition and abolish cortical plasticity associated with motor skill learning. Neuron 38:819-829.

Curran-Everett D (2000) Multiple comparisons: philosophies and illustrations. Am J Physiol Regul Integr Comp Physiol 279:R1-R8.

Decety J, Grezes J, Costes N, Perani D, Jeannerod M, Procyk E, Grassi F, Fazio F (1997) Brain activity during observation of actions. Influence of action content and subject's strategy. Brain 120:1763-1777.

Edwards MG, Humphreys GW, Castiello U (2003) Motor facilitation following action observation: a behavioural study in prehensile action. Brain Cogn 53:495-502.

Fadiga L, Fogassi L, Pavesi G, Rizzolatti G (1995) Motor facilitation during action observation: a magnetic stimulation study. J Neurophysiol 73:2608-2611.

Fadiga L, Fogassi L, Gallese V, Rizzolatti G (2000) Visuomotor neurons: ambiguity of the discharge or 'motor' perception? Int J Psychophysiol 35:165-177.

Fogassi L, Gallese V, Buccino G, Craighero L, Fadiga L, Rizzolatti G (2001) Cortical mechanism for the visual guidance of hand grasping movements in the monkey: a reversible inactivation study. Brain 124:571-586.

Fogassi L, Ferrari PF, Gesierich B, Rozzi S, Chersi F, Rizzolatti G (2005) Parietal lobe: from action organization to intention understanding. Science 308:662-667.

Gallese V, Goldman A (1998) Mirror neurons and the simulation theory of mind-reading. Trends Cogn Sci 2:293-501. 
Gallese V, Fadiga L, Fogassi L, Rizzolatti G (1996) Action recognition in the premotor cortex. Brain 119:593-609.

Gangitano M, Mottaghy FM, Pascual-Leone A (2001) Phase-specific modulation of cortical motor output during movement observation. NeuroReport 12:1489-1492.

Grafton ST, Arbib MA, Fadiga L, Rizzolatti G (1996) Localization of grasp representations in humans by positron emission tomography. 2. Observation compared with imagination. Exp Brain Res 112:103-111.

Grezes J, Decety J (2002) Does visual perception of object afford action? Evidence from a neuroimaging study. Neuropsychologia 40:212-222.

Hari R, Forss N, Avikainen S, Kirveskari E, Salenius S, Rizzolatti G (1998) Activation of human primary motor cortex during action observation: a neuromagnetic study. Proc Natl Acad Sci USA 95:15061-15065.

Heyes CM, Foster CL (2002) Motor learning by observation: evidence from a serial reaction time task. Q J Exp Psychol A 55:593-607.

Horn RR, Williams AM, Scott MA (2002) Learning from demonstrations: the role of visual search during observational learning from video and point-light models. J Sports Sci 20:253-269.

Iacoboni M, Woods RP, Brass M, Bekkering H, Mazziotta JC, Rizzolatti G (1999) Cortical mechanisms of human imitation. Science 286:2526-2528.

Iacoboni M, Molnar-Szakacs I, Gallese V, Buccino G, Mazziotta JC, Rizzolatti G (2005) Grasping the intentions of others with one's own mirror neuron system. PLoS Biol 3:e79.

Järveläinen J, Schürmann M, Hari R (2004) Activation of the human primary motor cortex during observation of tool use. NeuroImage 23:187-192.

Jeannerod M (1994) The representing brain: neural correlates of motor intention and imagery. Behav Brain Sci 17:187-245.

Kakei S, Hoffman DS, Strick PL (1999) Muscle and movement representations in the primary motor cortex. Science 285:2136-2139.

Kakei S, Hoffman DS, Strick PL (2001) Direction of action is represented in the ventral premotor cortex. Nat Neurosci 4:1020-1025.

Karni A, Meyer G, Jezzard P, Adams MM, Turner R, Ungerleider LG (1995) Functional MRI evidence for adult motor cortex plasticity during motor skill learning. Nature 377:155-158.

Kleim JA, Barbay S, Nudo RJ (1998) Functional reorganization of the rat motor cortex following motor skill learning. J Neurophysiol 80:3321-3325.

Maeda F, Kleiner-Fisman G, Pascual-Leone A (2002) Motor facilitation while observing hand actions: specificity of the effect and role of observer's orientation. J Neurophysiol 87:1329-1335.

Mattar AG, Gribble PL (2005) Motor learning by observation. Neuron 46:153-160.

Mitz AR, Godschalk M, Wise SP (1991) Learning-dependent neuronal activity in the premotor cortex: activity during the acquisition of conditional motor associations. J Neurosci 11:1855-1872.

Nishitani N, Hari R (2000) Temporal dynamics of cortical representation for action. Proc Natl Acad Sci USA 97:913-918.

Nishitani N, Hari R (2002) Viewing lip forms: cortical dynamics. Neuron 36:1211-1220.

Oldfield RC (1971) The assessment and analysis of handedness: the Edinburgh inventory. Neuropsychologia 9:97-113.

Pascual-Leone A, Nguyet D, Cohen LG, Brasil-Neto JP, Cammarota A, Hallett M (1995) Modulation of muscle responses evoked by transcranial magnetic stimulation during the acquisition of new fine motor skills. J Neurophysiol 74:1037-1045.
Patuzzo S, Fiaschi A, Manganotti P (2003) Modulation of motor cortex excitability in the left hemisphere during action observation: a single- and paired-pulse transcranial magnetic stimulation study of self- and nonself-action observation. Neuropsychologia 41:1272-1278.

Pavlides C, Miyashita E, Asanuma H (1993) Projection from the sensory to the motor cortex is important in learning motor skills in the monkey. J Neurophysiol 70:733-741.

Petrosini L, Graziano A, Mandolesi L, Neri P, Molinari M, Leggio MG (2003) Watch how to do it! New advances in learning by observation. Brain Res Brain Res Rev 42:252-264.

Rizzolatti G, Craighero L (2004) The mirror-neuron system. Annu Rev Neurosci 27:169-192.

Rizzolatti G, Fadiga L (1998) Grasping objects and grasping action meanings: the dual role of monkey rostroventral premotor cortex (area F5). Novartis Found Symp 218:81-95; discussion 95-103.

Rizzolatti G, Fadiga L, Gallese V, Fogassi L (1996) Premotor cortex and the recognition of motor actions. Brain Res Cogn Brain Res 3:131-141.

Rizzolatti G, Fogassi L, Gallese V (2001) Neurophysiological mechanisms underlying the understanding and imitation of action. Nat Rev Neurosci 2:661-670.

Rossini PM, Barker AT, Berardelli A, Caramia MD, Caruso G, Cracco RQ, Dimitrijevic MR, Hallett M, Katayama Y, Lucking CH, Maertens de Noordhout AL, Marsden CD, Murray NMF, Rothwell JC, Swash M, Tomberg C (1994) Non-invasive electrical and magnetic stimulation of the brain, spinal cord and roots: basic principles and procedures for routine clinical application. Report of an IFCN committee. Electroencephalogr Clin Neurophysiol 91:79-92.

Rothwell JC, Traub MM, Day BL, Obeso JA, Thomas PK, Marsden CD (1982) Manual motor performance in a deafferented man. Brain 105:515-542.

Sanes JN, Donoghue JP (2000) Plasticity and primary motor cortex. Annu Rev Neurosci 23:393-415.

Shimazu H, Maier MA, Cerri G, Kirkwood PA, Lemon RN (2004) Macaque ventral premotor cortex exerts powerful facilitation of motor cortex outputs to upper limb motoneurons. J Neurosci 24:1200-1211.

Stefan K, Duque J, Mazzocchio R, Sawaki L, Ungerleider L, Classen J, Cohen LG (2003) Encoding a motor memory by action observation. Soc Neurosci Abstr 29:77.7.

Strafella AP, Paus T (2000) Modulation of cortical excitability during action observation: a transcranial magnetic stimulation study. NeuroReport 11:2289-2292.

Sundara M, Namasivayam AK, Chen R (2001) Observation-execution matching system for speech: a magnetic stimulation study. NeuroReport 12:1341-1344.

Vinter A, Perruchet P (2002) Implicit motor learning through observational training in adults and children. Mem Cognit 30:256-261.

Vogt S (1995) On relations between perceiving, imagining and performing in the learning of cyclical movement sequences. Br J Psychol 86:191-216.

Werhahn KJ, Fong JK, Meyer BU, Priori A, Rothwell JC, Day BL, Thompson PD (1994) The effect of magnetic coil orientation on the latency of surface EMG and single motor unit responses in the first dorsal interosseous muscle. Electroencephalogr Clin Neurophysiol 93:138-146. 